\title{
Insole Systems for Disease Diagnosis and Rehabilitation
}

This paper was downloaded from TechRxiv (https://www.techrxiv.org).

\section{LICENSE}

CC BY 4.0

SUBMISSION DATE / POSTED DATE

19-02-2022 / 23-02-2022

\section{CITATION}

Gao, Shuo; Zhang, Zhiyuan; Wang, Jiamu; Xu, Zhenyu; Grimaldi, Nicolas; Zhao, Mufan; et al. (2022): Insole Systems for Disease Diagnosis and Rehabilitation. TechRxiv. Preprint.

https://doi.org/10.36227/techrxiv.19200425.v1

$\mathrm{DOI}$

10.36227/techrxiv.19200425.v1 


\title{
Insole Systems for Disease Diagnosis and Rehabilitation
}

\author{
Shuo Gao, Zhiyuan Zhang, Zhenyu Xu, Nicolas Grimaldi, Jiamu Wang, Mufan Zhao, Ruilin Pang, \\ Yueming Sun, Yanning Dai, Hu Boyi
}

\begin{abstract}
Neurological and orthopedic chronic diseases are proven to numerous complications and related dysfunction for patients, and are leading factors of death and disability. Conventional methods of diagnosis and rehabilitation are conducted at hospitals. However, due to the aging society and controversial privacy protection, traditional methods implemented in medical institutions have faced numerous challenges. Hence, internet of healthcare things (IoHT) based techniques have recently begun to be implemented. In order to provide flexible utilization and secure privacy for patients, wearable devices, like whole body devices based on electromyography (EMG) and radio frequency identification (RFID) insole systems, based on gait-analysis were applied. Among these techniques, insole systems for medical analysis are more suitable, because the root cause of neurologic and orthopedic chronic diseases are directly related to several gait characteristics. Neurologic and orthopedic chronic diseases are caused by lesions of the central nervous system (CNS), peripheral nerves (PN) and orthopedic limbs, similarly gait features are comprehensive results generated by the coordination of CNS, PN and lower limbs. However, owing to lack of standards for instrumentation and the knowledge gap between engineering and pathogenic mechanism, the medical application of insole systems and the development of IoHT are hindered. This article is presented to address these issues. In this context, five plantar sensing mechanisms and their related products will be overviewed. Next, pathogenic mechanisms and pathological gait features of six representative chronic neurologic diseases will be displayed. Likewise, the development of corresponding insole systems for supervision and recovery for these diseases will be reviewed as well. Finally, challenges and future trends for current work will be discussed. This article will not only provide guidelines for the design of insole systems
\end{abstract}

Shuo Gao is with the School of Instrumentation and Optoelectronic Engineering, Beihang 100091 China (e-mail: shuo_gao@buaa.edu.cn).

Zhiyuan Zhang is with the School of Instrumentation and Optoelectronic Engineering, Beihang 100091 China (e-mail: exzzy32@163.com).

Zhenyu Xu is with the School of Instrumentation and Optoelectronic Engineering, Beihang 100091 China (e-mail: xuzhenyu_buaa@qq.com).

Nicolas Grimaldi is with the school of J. Crayton Pruitt Family Department of Biomedical Engineering

Jiamu Wang is with the School of Transportation Science and Engineering, Beihang 100091 China (e-mail: xuzhenyu_buaa@qq.com).

Mufan Zhao is with the School of Artificial Intelligence, Beihang 100091

China (e-mail: zhaomufan15@foxmail.com).

Ruilin Pang is with the School of Automation Science and Electrical

Engineering, Beihang 100091 China (e-mail: 1378632329@qq.com). Yueming Sun is with the School of Electronics and information engineering, Beihang 100091 China (e-mail: 13552875745@163.com).

Yanning Dai is with the School of Instrumentation and Optoelectronic

Engineering, Beihang 100091 China (e-mail: 16171056@buaa.edu.cn).

Boyi Hu is with the school of Industrial and Systems Engineering targeted for specific diseases but will also inspire the innovation of novel ideas.

Index Terms - gait analysis, neurological disease, plantar pressure, insole systems

\section{INTRODUCTION}

$\mathrm{T}$ HE branches of chronic diseases are various, and include neurologic, orthopedic and musculoskeletal diseases. Their clinical manifestations, like motor impairment and tissue damage, can lead to death and disability of old people [1]. Features of these diseases can be: asymptomatic, pose long incubation periods, multiple pathogens and implicate numerous complications as well as related dysfunctions. Thus, effective methods of diagnosis and rehabilitation for these diseases are in high demand. Conventionally, the rehabilitation and diagnosis for patients of chronic diseases are conducted at hospitals. Medical professionals will give feasible prescriptions supported by methods like medical assays and X-ray films.

However, with the fast development of aging society boosting the number of senior people, more orthopedic and neurologic chronic patients arise, which places heavy burdens to medical institutions and staff [3]. Thus, the need for more flexible and remote medical methods has risen. Therefore, novel recovery methods based on internet of healthcare things (IoHT) have emerged in past few decades. IoHT refers to the application of network systems as a way of connecting patients and their medical data to available healthcare resources (i.e., hospitals, doctor, nurses, etc.) [2].

Many IoHT based techniques allow patients to carry out consultations online [4]. Currently, these techniques can be attained through two main methods: computer vision (CV) based methods and wearable devices.

The CV method utilizes vision-based monitoring means to establish active and assisted living medical models of patients online. Through analyzing parameters obtained, medical staff can provide an effective diagnosis. The representative application of this method can be classified into external type and internal type according to the position of the monitoring devices.

The former type focuses on using video-based devices, like RGB-D sensors, inside a room to illustrate silhouette models of patients and their environment. As a result, gestures and movements recorded can be evaluated by medical staff [124].

As for the latter one, the internal kind, Wireless Capsule Endoscopy (WCE) is one example of a representative 


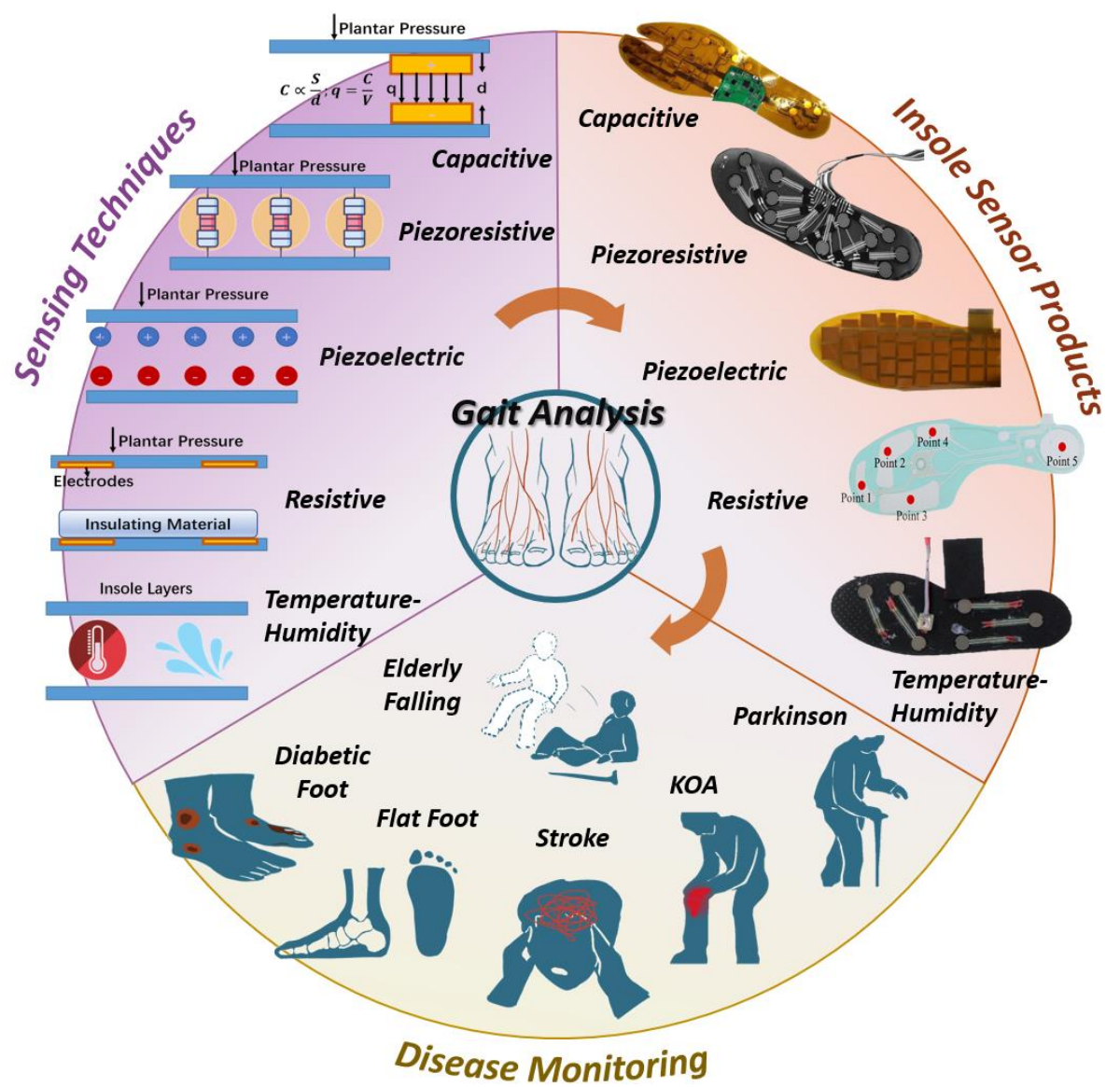

Fig. 1. demonstration of diseases; sensing techniques and current products

technique. Through placing a capsule-like camera inside human body, the camera will record and transmit videos and images of patients for medical analysis [125].

These applications contribute to precise and considerable diagnosis through medical imaging. However, the use of wholebody data and camera-based environments has raised concerns about privacy and complex utilization has also emerged. As a result, privacy of users might be invaded while using medical methods above.

Considering this dilemma and the concerns of patients and medical staff, wearable device-based supervision and diagnosis for rehabilitation has become a more suitable method. This could be attributed to two main reasons: more flexible utilization and better privacy protection.

The former one is based on real-time vital sign transmission of wearable equipment, which can enable medical staff to monitor the human body and remotely evaluate health status basing on ample data [5]. Besides, patients can execute parameter detection and collection in daily activities. Additionally, due to the miniscule volume and small mass of the wearable sensors, patients will be undisturbed most of time. As for the latter, conventional methods focus on sharing medical information to establish personalized healthcare systems, but the cyberinfrastructure boundaries are not specified to prevent private data leakage [6]. Unlike conventional ways, wearable devices are fixed only at targeted places, thus they merely extract key parameters from specific regions which are related to lesions. This way, patient privacy of whole-body data will not be invaded, while still maintaining effective supervision and diagnosis.

Nowadays, a variety of current wearable devices are available. They can be classified into whole-body devices and insole systems. The former type consists of techniques like electromyography (EMG) and radio frequency identification (RFID), while gait-analysis is a representative technique of the latter type.

The EMG technique aims to extract electric signals from neuromuscular activities. Physically, through sticking electrodes (e.g., dry electrodes, gelled electrodes, and sensors) onto human skin, myoelectric signals are obtained [10]. Signals obtained will be analyzed by double-threshold methods [11] to diagnose and evaluate the current status of patients.

As for the RFID, it is conventionally composed of tags, readers, antennas and related software [12]. Although tags are attached to the skin of patients and readers are distributed to different locations in the body, medical staff can nonetheless retrieve the data from tags through mobile devices like Bluetooth sensors. RFID is widely applied in drug tracking, patient verification and supervision [13].

Apart from the techniques mentioned above, insole systems for rehabilitation and diagnosis can be obtained mainly by gait analysis, which is more suitable for analyzing diseases. The most important reason is that neurologic chronic diseases, like 


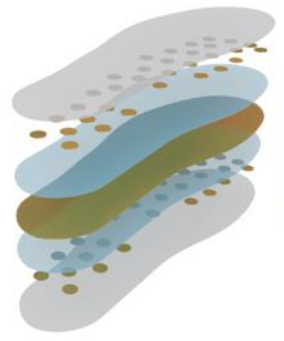

(a)

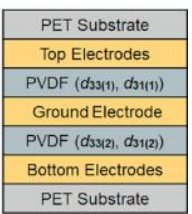

PET Substrate

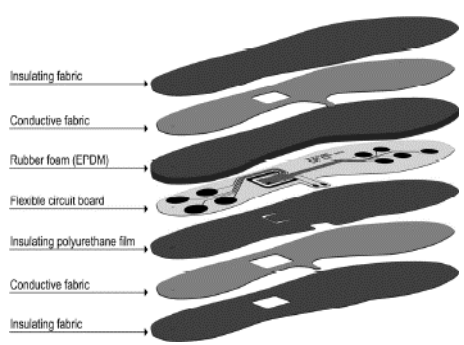

(c)

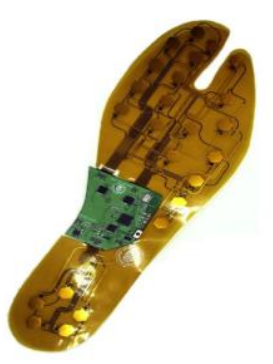

(d)

Fig.2 (a) structure of piezoelectric insole systems [130]; (b) piezoelectric prototypes [129]; (c) structure of capacitive insole systems [131]; (d) prototypes of capacitive insole systems [128]

Parkinson's disease (PD) and cerebrovascular accidents (strokes), are caused by lesions of the central nervous system (CNS) and the peripheral nerves (PN) [7]. Besides, typical orthopedic diseases like flat foot are comprehensive results from lower limbs [7]. Significantly, gait features can comprehensively reflect and demonstrate the coordination of CNS, PN and lower limbs [8]. Hence, through analyzing these gait patterns, related results, like plantar pressure distribution, can contribute to orthopedic and neurologic lesion monitoring, like foot ulceration and loss of dopamine [9], to provide ample information for recovery.

Present insole systems, including prototypes produced by institutions and commercial systems like F-scan and Pedar-X, all guarantee the accuracy of their measurements. But several factors hinder their medical application. On one hand, there is a lack of standards for instrumentation influencing the design process. For instance, while designing systems for the diagnosis of diabetes, desired parameters or sensing mechanisms are not stipulated. This issue delays the promotion of systems. Besides, the knowledge gap between medical professionals and gait researchers has not been bridged effectively yet, which reduces the efficiency in coordination and the utilization of products.

Under the current dilemma, this essay is presented to tighten the gap between gait analysis and disease diagnosis and recovery from three aspects. Firstly, this essay summarizes five traditional sensing mechanisms and presents key parameters (measuring range, sensors type and virtues) of some current products. These options can be used by researchers when conducting gait-based medical research. As shown in Fig.1, this essay focuses on relationship between the utilization of sensing techniques and chronic diseases.

Secondly, the pathogenic mechanism and pathological gait features of six representative chronic neurologic diseases are displayed. Based on the explanation of lesions and their corresponding gait parameters, researchers can obtain more guidelines while designing novel insole systems targeted for specific diseases. Furthermore, the history of the development of the relationships between the six neurologic chronic diseases and their corresponding insole systems for diagnosis and rehabilitation is described in detail. Specifically, this section reviews the methods and effects of gait analysis-based rehabilitation.

Finally, this article discusses the black-box issue, which hinders the development of IoHT and generates troubles in diagnosis and rehabilitation. Afterwards, we propose the integration of digital twin (DT) models with current work as a potential trend in future advancements, which will inspire more colleagues to conduct further research.

This article is structured as follows: section II overviews five conventional plantar pressure sensing techniques. Section III reviews the development of insole sensor systems for six specific disease diagnoses and recovery supervisions in detail. Parameters of healthy patients and patients with diseases are also compared in several tables. Effects of rehabilitation means are demonstrated in several tables as well. Section IV firstly discusses black-box predicament of current products, then proposes DT models to achieve data, physical and model fusion in hopes of improving interpretability of gait analysis for disease monitoring.

\section{LITERATURE REVIEW ABOUT MAINSTREAM DETECTING METHODS}

Insole sensors are designed for the purpose of obtaining temporal and spatial gait parameters like cycle time, swing and stance phase, step and stride length, and pressure distribution. The goal can be conventionally attained through plantar force sensing mechanisms which consist of piezoresistive, resistive, capacitive, piezoelectric, and temperature-humidity means as shown in Fig.1.

In following paragraphs, we overview seven distinct insole pressure sensing mechanisms and their current products. The illustrations of techniques and prototypes are demonstrated in Fig.2-4.

\section{A. Piezoresistive techniques}

The principle of this technique revolves around obtaining relationships between applied stress and altered electric resistance of a material. Plantar stress detection can be attained through two conventional ways: adhesive-method and screenprinting technology.

Adhesive-method involves fixing separate force sensing resistors (FSR) on special regions of the insole according to symptoms of diseases. For instance, the insole system for PD place 16 FSRs at forefoot and heel [14]. Finally, this system is enclosed in shrink-wrap to avoid displacement and motioninduced abrasion.

Drawbacks of adhesive insole systems, like sensors overlap, friction and unstable mechanical capacity, can be addressed by 


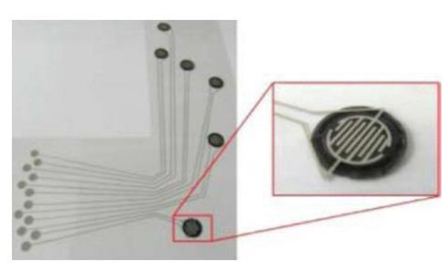

(a)

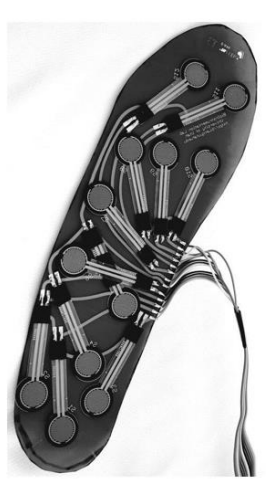

(b)

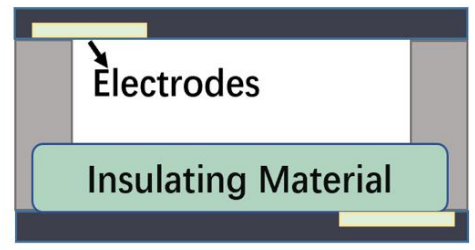

(c)

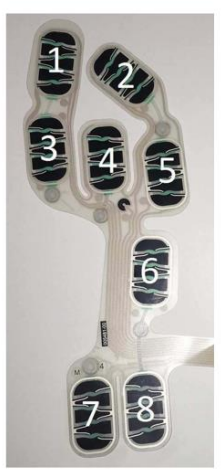

(d)

Fig.3 (a) elements of piezoresistive insole systems; (b) prototypes of piezoresistive insole systems [101] (c) elements of resistive insole systems [95]. (d) prototypes of resistive insole systems [95];

whole-piece design and fabrication, which are based on screen printing technology. Therefore, some commercialized products like the F-scan ${ }^{\circledR}$ system [15], which has a sensing pressure in the range of $7 \mathrm{kpa}$ to $1043 \mathrm{kpa}$; the multiwalled carbon nanotube -polydimethylsiloxane based sensors array [16], and the SurroSense Rx Insole produced by company Orpyx SI are widely utilized. Further improvements focus on shear stress detection. One system proposed can measure normal stress up to $400 \mathrm{kpa}$ and shear stress up to $80 \mathrm{kpa}$ [17].

Virtues like high-voltage sensitivity and low price diversify piezoresistive sensors' application. But hysteresis and creep issues occur in utilization, which require frequent calibration. Therefore, this technique is more suitable for short-term diseases instead of long-lasting therapy and monitoring.

\section{B. Resistive techniques}

These techniques are fulfilled through formulating relationships between resistance, element geometry, and structure. Traditionally, resistive force sensing can be achieved through contact surfaces and strain gauges.

The former method utilizes two resistive layers isolated by bracing frames, the imposed load will extrude them to approach. When in contact, current flows through the layers. The contacted area, which is correlated to an extern al force, can reflect the force through resistance. Previous work [18] presents an insole system for diabetic patients in real time tracking. The contact area between layers will surge as applied force increases.

The latter mean is conducted by attaching strain gauges to mechanical structures that will measure strain when pressed. The strain of resistive material will also give rise to a change of resistance. For instance, past work [19] has proposed a mechanical insole composed of strain gauge load cells and multiple-axis force sensors. The system can provide shear load and normal load synchronously.

Some highly-integrated resistive and piezoresistive insole systems are produced by Tek-scan company, as illustrated in Fig. 2.

Resistive-based insole systems can contribute to long-term tracking because of its low unrepeatability [18] and low cost. However, its virtues do not include simple structures, which indicates that systems may have bigger volumes. Furthermore, its complicated manufactures will suffer from massive durability problems, and result in less users.

\section{Capacitive techniques}

As capacitance is significantly influenced by the dielectric constant, capacitive sensors are sensitive to environmental humidity and electromagnetic interference. Typically, capacitive sensors are constructed with an elastomer medium and two electrode layers. The applied load will influence the capacitance through changing the distance between upper and lower layers; consequently, plantar stress can be obtained by measuring capacitance.

Current products can be classified into experimental systems and commercial systems.

One experimental study presents an integrative method [20]. This work utilizes sensor layers with fixed capacitance arrays, as well as a flexible circuit layer is also added to the integrated in-shoe system. This system is suitable for stroke patients' rehabilitation progress.

Commercial capacitive insole products [21][22] focus on durability and long-term utilization, thus they can be applied for patient tracking. One existing product obsess prolong working lifetime, it can support $100 \mathrm{~km}$ running while the extreme measurable pressure is $400 \mathrm{kpa}$; thus, it is suitable for examining the weight-supporting gait pattern after extremity fractures [21]. Meanwhile, a product based on Pedar-X [22] can support sustainable and continuous utilization, therefore it is widely exploited in diabetic foot ulcers [24] and ankle osteoarthritis (AO) [25] diagnosis because these diseases both require longtime data recording for prediction. Open-go capacitive insole systems (illustrated in Fig.3), are produced by Moticon company, they are also available for diabetic foot diagnosis and supervision.

Sustained working lifetime and dynamic performance are virtues of capacitive sensors, but the limited force sensing range should be considered when choosing this technique. 


\section{Piezoelectric techniques}

Piezoelectric techniques are based on electrical charges, which accumulate on the surface of non-central symmetric materials when external force is applied.

To utilize this technique, piezoelectric insole sensors are usually integrated as electrode/piezoelectric film/electrode sandwiched structures; it possesses virtues like high sensitivity, low power consumption and a simple structure.

A certain prototype [23], is designed for diabetes diagnosis in its early stages. Its 8 separate sensors with rigid substrates structure can reduce negative impacts of crosstalk from other axials. In addition, one essay [24] demonstrates that flexible printed circuit boards serve as adequate electrode layers for mass production.

Another merit of piezoelectric sensors is their ability to detect force in different directions. This owes to the fact that the piezoelectric effect can occur on both vertical and parallel axes to the film surface, and the force-to-voltage coefficient depends on strength and polarization directions. Past work [25] attains this through utilizing a transducer alongside four independent piezoelectric film sensors.

However, polarization orientation suffered by piezoelectric materials cannot be aligned in the same direction, thus will arise a response from unwanted force.

Besides, their drawbacks also include leakage generated in the subsequent amplifying circuit which indicates they cannot measure static force. For all the reasons mentioned above, piezoelectric-based sensors have not been commercialized yet.

\section{E. Temperature and humidity-based techniques}

Patients with peripheral neuropathies have risks suffering lower extremity skin breakdown [27,28], which could be indicated by temperature and moisture [29,30]. Medical professionals thus propose insole and skin temperature measuring methods for inflammation and tissue damage detection, both of which are caused by repetitive stress [31,32].

Former methods to support insole temperature measurement are mostly based on thermistors, in which the deviation of resistance can reflect the temperature change. For instance, three thermistors at $5 \mathrm{k}$ are fixed at heel, big toe and ball of plantar. But the sensitivity is limited, only differences greater than $2.2^{\circ} \mathrm{C}$ can be observed [34].

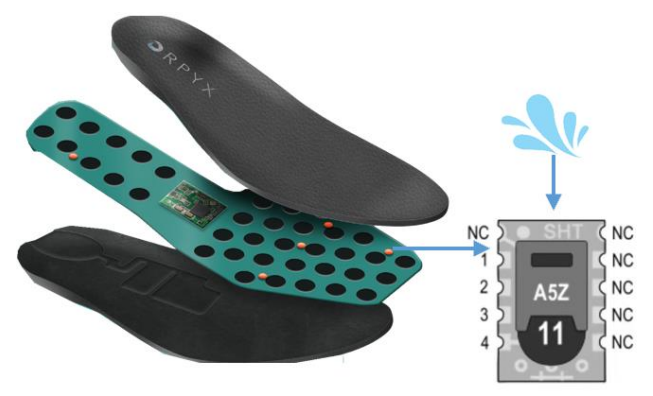

Fig.4 temperature-humidity based insole systems and inside elements

To uplift the sensitivity, more integrated sensors have been produced in the last decade. The SHT series produced by company Sensirion [26,33] and DHT series produced by Aosong Electronics company [35] series of temperature sensors are widely used. A SHT-21 module is fixed on a printed circuit board (PCB). The sensitivity integrated system can attain $4 \%$ and $0.3^{\circ} \mathrm{C}$, when the fluctuation of temperature is less than $15^{\circ} \mathrm{C}$ [32]. Similarly, a SHT-11 module is integrated with an amplifier and A/D converter in a tiny chip. Results shows that the sensitivity is $3 \%$ and $0.4^{\circ} \mathrm{C}$ [33]. They are both suitable for the diagnosis and supervision of foot skin related lesions, like diabetic ulcers.

A representative insole system (shown in Fig.3) produced by Orpyx company can measure temperature and pressure at same time, thus provide more comprehensive data for diagnosis.

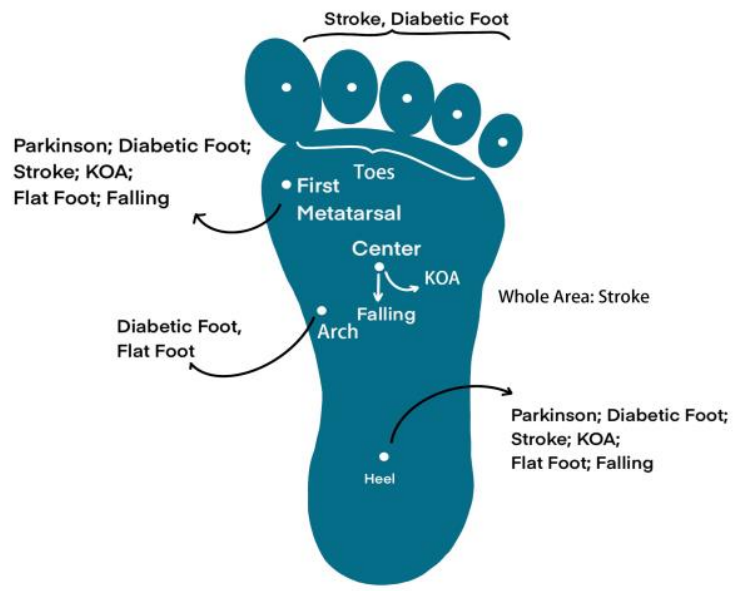

Fig. 5. Sensing points for chronic diseases

\section{F. Virtues and drawbacks of techniques}

The virtues and drawbacks of techniques makes them suitable for diverse diseases. Piezoresistive and resistive methods both have a high force sensing range and low price, but they suffer from low repeatability and the influence of temperature and humidity. Temperature and humidity-based sensors have low energy consumption and cost, but their inability to detect plantar pressure limit their gait related application. As for capacitance and piezoelectric methods, high sensitivity of force is their most significant merit, while the sensitivity to temperature and electromagnetic interference change are their main drawbacks.

TABLE I

GAIT PARAMETERS OF PD PATIENTS AND HEALTHY PEOPLE

\begin{tabular}{ccc}
\hline Parameters & Patients & Healthy people \\
\hline Gait velocity $(\mathrm{m} / \mathrm{s})$ & & Higher \\
Gait cycle (s/step) & Higher & Higher \\
Stride length (m) & & \\
Stride phase duration (s) & Higher & \\
Stance phase duration (s) & Higher & \\
Double support phase duration $(\mathrm{s})$ & Higher & \\
Peak plantar pressure (kPa) & & Higher \\
Plantar pressure central frequency $(\mathrm{Hz})$ & Higher & \\
\hline
\end{tabular}




\section{SIX CHRONIC DISEASES AND CORRESPONDING INSOLE SYSTEMS}

Based on the techniques above, fruitful insole systems have been fabricated. Their precise data (plantar pressure and humidity) can contribute to the calculation of temporal and spatial gait parameters (gait frequency, gait velocity, step length and duration phase and so on), which in turn aid in rehabilitation and diagnosis for patients of various diseases. Their more detailed information and parameters will be present in the table in appendix, which can help more colleagues to choose suitable insole systems and sensors.

In this section, two kinds of neurologic diseases (PD and stroke), two kinds of orthopedic diseases (knee osteoarthritis and flat foot), two kinds of musculoskeletal diseases (diabetes and elderly-falling) and their corresponding insole systems will be introduced separately. Besides, the sensing points of each diseaeses are illustrated in Fig.5. The pathogenic demonstrations of chronic diseases are shown in Fig.6.

\section{A. Parkinson}

PD is a common neurodegenerative disease among middleaged and senior people [36]. Patients' dopamine-producing cells will gradually degrade, which blocks the nerve cells controlling muscle movement, and give rise to disturbances in motion (e.g., freezing of gait (FoG), slow movement, and postural instability [37]). Medical treatments for PD concentrate on dopamine replacement or surgery, whose postoperative responses suffers from individual differences and unpredictable fluctuations [38]; hence, highlighting the importance of prognosis tracking. To provide effective postoperative monitoring and treatment methods for PD patients, various wearable devices based on gait analysis have been developed in previous studies. Their concerned parameters include gait velocity, stride length, gait phases duration, and plantar pressure distribution; typical values for PD patients and healthy people are summarized in Table 1 [39, 67, 68].

Research of wearable insole systems for PD are divided into two parts: parameters monitoring and external rhythmical cues stimulation therapy [40]; each part will be discussed in this section.

Sensors for PD's parameter measuring can be categorized into inertial sensors (i.e., gyroscope and accelerometer) $[45,46]$ and plantar pressure sensors [41, 42, 47, 127]. For the former category, R. Hua et al. [45] proposed a PD monitoring insole contains two accelerometers, which are placed at the heel and the first metatarsal of the plantar. The vertical component of the measured acceleration is used to detect gait events and phase duration, while the horizontal component is applied to calculate gait velocity and stride length. The detection accuracy achieved $88.74 \%$ on average. Moreover, the insole system could differentiate patients" "activity state" and "inactivity state", and thus change the system's working mode to save battery power. B. Jens et al. [46] attached an IMU sensor at the lateral heel area outside a shoe to recognize the gait impairment degree of PD patients. Gait features in both time and frequency domains are extracted, and a linear discriminant analysis (LDA) model is used for classification. Results showed that the gait cycle, movement range, and signal energy in the frequency domain $(0.5-3 \mathrm{~Hz})$ are the most suitable indicators of PD-impaired gait.

The plantar pressure sensors are applicable as well. K. Grandez et al. [41] proposed an insole system with 3 FSRs distributed at the forefoot and heel areas, to compare the gait parameters between PD patients and healthy people. The obtained signals are visualized through a PC application and then analyzed by doctors. Results showed that patients with PD tend to have a higher force central frequency (around $1.8 \mathrm{~Hz}$ ) than healthy people (around $0.8 \mathrm{~Hz}$ ), and the patients' plantar peak pressure amplitude is $40 \%$ lower, approximately.

In terms of adjuvant therapy, wearable devices are used to impose external stimulation cues on patients. The mainstream external cues can be divided into three categories: visual, auditory, and somatosensory. For visual cues, the laser-assisted device (LAD)-based methods are widely used. For instance, E. Lim et al. [70] used a hand-held LAD as a walking aid for PD patients. When the patients encounter FoG or hesitation, the LAD will project a meter-wide red line ahead for instructions. After two days of training, the gait cycles of 3 patients were reduced from approximately $10 \mathrm{~s} / \mathrm{step}$ to less than $5 \mathrm{~s} / \mathrm{step}$

TABLE II

TYPICAL GAIT PATTERNS OF STROKE PATIENTS

\begin{tabular}{ccc}
\hline Gait features & $\begin{array}{c}\text { Affected } \\
\text { lower extremity }\end{array}$ & $\begin{array}{c}\text { Unaffected } \\
\text { lower extremity }\end{array}$ \\
\hline $\begin{array}{c}\text { Swing phase (\%) } \\
\text { Stance phase (\%) }\end{array}$ & higher \\
Single support phase (\%) & higher & \\
Double support phase (\%) & higher & \\
Ground reaction force (N) & & higher \\
Peak Pressure (kPa) & & higher \\
experimentally. & \\
Delivering auditory stimulation is another option. In previous
\end{tabular}

work [48], E. Jovanov et al. developed a therapy system with a stimulation headset and an on-shoe inertial sensor. The inertial sensor detects the FoG events in real-time through the signal energy distribution pattern in the frequency domain, and then the headset plays the rhythmic auditory recording (e.g., 'click' sound) to unfreeze patients' gait. The system is able to provide effective therapy within 580ms latency. Similarly, Marc Bachlin et al. [49] utilized an earphone and three on-body acceleration sensors (placed at ankle, thigh, and waist) to detect and treat FoG. Detection sensitivity of $73.1 \%$ was finally achieved in 237 tests. Visual and auditory external methods make therapy and diagnosis more effective; however, both of them suffer from environmental limitations. Acoustic cues such as music beats or metronomes might be disturbed by noise, while visual-based methods highly depend on natural lighting conditions [50]. To address these issues, somatosensory rhythmic external cue-based systems are proposed. For instance, past work [69] demonstrated a step-synchronized vibration system with three vibratory devices fixed at high-pressure insole areas (heel and metatarsus). The vibratory motors are activated when the foot is in contact with the ground. Results revealed that patients' cycle, step length, and gait velocity can be improved, with a coefficient of variation (CV) at around 0.05 . 
The research mentioned above not only provides adequate evidence for diagnosis, but also uplift surgery rehabilitation by contributing to PD treatment.

\section{B. Diabetes}

Diabetes is a group of metabolic diseases characterized by hyperglycemia. With the improvement of living standards, the incidence of diabetes has been continuously increasing in the past few decades. The International Diabetes Federation warned in 2005 that one in ten people will have diabetes by 2030 [63]. Diabetes can cause many complications, among which diabetic foot is the most serious one. If not treated in time, it will require amputation as it is a life-threatening condition [51]. Diabetic foot has two notable pathological symptoms: inflammation and dryness. Inflammation warns that the tissues in the area have been ulcerated, while dryness warns the cessation of the autonomic nervous function [52, 53].

Early-stage research of diabetic foot focused on foot ulcer detection. In 1986, M. E. Edmonds et al. [72] found that diabetic foot ulcers occur more often at the forefoot and toes than other plantar areas. Then in 1998, D. G. Armstrong et al. [71] revealed a close association between high plantar pressure $(\geq$ $60 \mathrm{kPa}$ ) and ulceration in the diabetic population. A recent study [55] demonstrated that patients with foot ulcers tend to have a foot rolling motion in the mid-stance phase during walking. Based on the medical results, insole systems for plantar pressure measurement have been proposed for foot ulcer monitoring. For example, P. Aqueveque et al. [57] developed an insole system to measure the pressure at high-stress areas. Eight capacitive sensors, with diameters of $15 \mathrm{~mm}$, are placed at the toes, metatarsus, arch, lateral midfoot, and heel. Each sensor unit is able to bear weights up to $20 \mathrm{~kg}$. In another design [54], a micro-electronic mechanical smart portable system
(MEMS) was proposed based on piezoresistive techniques for the detection of foot ulcers. The sensor achieved a wide sensing range of 0-2 $\mathrm{MPa}$.

Moreover, recent studies aim to figure out the influence of individual patient differences on the pressure at the ulcer areas. M. J. Hessert et al. [64] pointed out that younger people and older people possess different plantar pressure features. The younger people experience less pressure on the lateral area of feet, which may improve body stability during walking. M. Nouman et al. [56] used the Pedar- $\mathrm{X}{ }^{\circledR}$ in-shoe pressure measurement system to analyze the relationship between the plantar pressure distribution pattern and patients' weight. As a result, the midfoot peak pressure of the obese group is significantly higher when compared with other patients. Therefore, a more accurate diagnosis of ulceration must consider additional physical factors of patients as well.

Apart from plantar pressure amplitude, foot temperature and humidity also contribute to diabetes foot detection, as they are closely related to foot dryness. T. Bernard et al. [34] devised an insole sensor system for the simultaneous pressure and temperature monitoring of diabetic feet. In the system, 3 thermistors and 3 pressure sensors were placed at the big toe, first metatarsal, and heel. Based on the comparative experiments between diabetic patients and healthy people, it is determined that plantar pressure above $60 \mathrm{kPa}$ and a temperature difference above $2.2^{\circ} \mathrm{C}$ (between different sensor locations) are representative of an early foot ulcer. M. Anouar et al. [60] designed a flexible insole with 3 coupled temperature/humidity SHT1X sensors and 8 inductive force sensors. The monitored data (including plantar pressure, humidity, and temperature) is shown to patients and doctors through a mobile phone application. Lastly, the danger level of

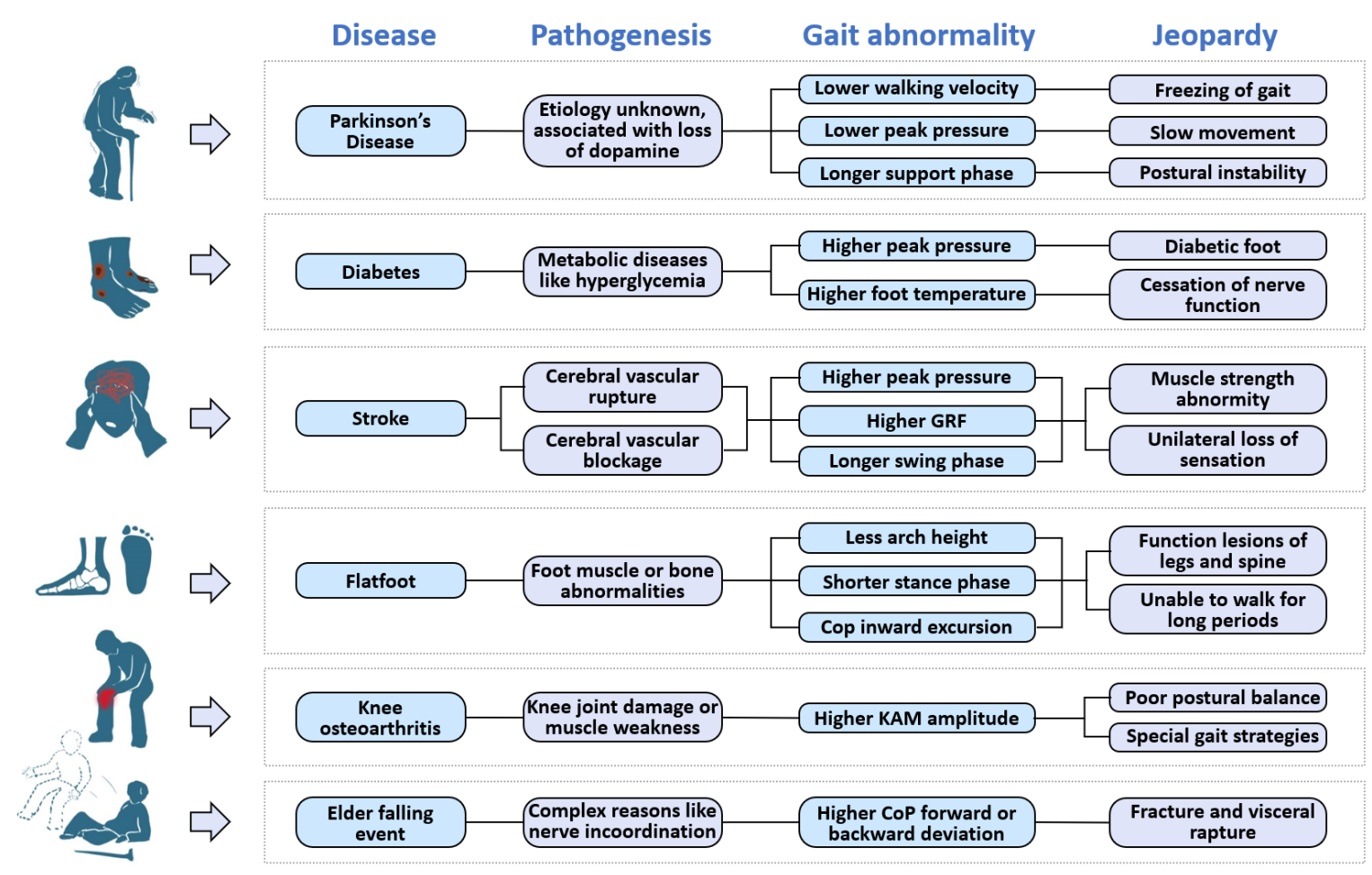

Fig. 6. Demonstrations of chronic diseases 
patients' foot ulcers is alerted in real-time through thresholdbased methods.

\section{Post-stroke Rehabilitation}

Stroke, an acute disease caused by cerebral blood vessels' rupture or blockage, is the prominent disabling causation for elderly people around the world [73,74]. Around $80 \%$ of poststroke patients suffer from different levels of motor impairments, such as muscle strength abnormity and the sensory loss of unilateral limbs [75]. To help patients recover motor functions and improve quality of life, efforts have been made in developing wearable kinematic assessment systems that monitor daily activities and help formulate clinical treatment plans.

The main concerning factor is the limb action symmetry during walking. In general, the affected side of lower extremity of stroke patients often generates relatively lower plantar pressure, and thus have longer stance phases, longer support phases, and lower plantar pressure amplitudes; these parameters are summarized in Table II [76, 77].

Various limb action monitoring systems have been reported for post-stroke rehabilitation. For instance, P. L. Meyer et al. [79] developed a shoe-based wearable system with 5 FSR sensors (located at the toes, metatarsus, and heel) to capture stroke-impaired gait phases. The sum of the detected pressure is used to recognize the moments of heel-strike and toe-off events, and thus calculate the duration of different phases. Based on experiments, which involved 16 healthy human subjects and 7 poststroke subjects, the system showed a 95\% confidence value for gait phases detection. A. M. Howell et al. [80] developed a 32-sensor insole to measure the ground reaction force and ankle moment of stroke patients. The ground reaction force was calculated by adding the force from each of the sensors; the ankle moment was calculated by multiplying the force of each sensor by its anterior-posterior distance to the ankle joint center. Results showed that patients suffering from an impaired gait had a noticeably different pattern in the signals energy concentration and peak numbers.

Moreover, to make insoles perfectly fit every patient in rehabilitation, L. Qin et al. [81] made innovations in the shape of the insole itself by producing 3D tailor-made insoles. Patients' feet were scanned to build 3D models in the software; then, the insole structures are simulated, and subsequently manufactured through silicone solution casting. Experimental results on patients showed that the measured peak pressure in the mid-foot area is higher than that of traditional flat insoles, which suggests a better contact between the insole and foot. This enabled a more customized and accurate rehabilitation for post-stroke patients.

M. M. Organero et al. [82] focused on optimizing the quantity and location of the sensors, which is a very important but often neglected section. An insole with 8 FSR sensors was used as the original template. However, a more cost-effective solution was attained by minimizing the L1 distance of plantar pressure patterns measured by the entire 8 sensors, and rather using a combination of 4 sensors (located at positions 1, 4, 6, and 7). Thus reducing 50\% of the system's cost, which is an important aspect for stroke survivor users and commercial companies. Apart from sensor layout, there are several other aspects worth optimizing. For example, in hardware design, the choice of utilizing economical and practical microchips remains less successful and still requires further research.

\section{Flatfoot}

Arch is an important structure of the human foot, which locks the midfoot joints and absorbs the impact forces from the ground, enabling steady support and propulsion of the human body [83]. Characterized by arch collapse, flatfoot is a common foot deformity caused by foot muscles or bones abnormalities. It prevents patients from long-time walking and other lower limb exercises. In the long term, it may even cause functional lesions in the leg and spine [84]. Therefore, the early detection of flatfoot is important for effective rehabilitation.

The clinical diagnosis of flatfoot is based on two kinds of information, as listed in Table III [86-88, 90, 91]. The first is the arch structure parameters, including height, width, and the contact area between the plantar and ground [90]; the second is gait features such as gait phases, center of pressure $(\mathrm{CoP})$, and ankle joints' rotation angles [85]. In previous studies, a number of methods and techniques have been used to measure these two kinds of parameters.

The conventional techniques mainly focus on plantar structure measurement. For example, in [86], an ultrasonic distance sensor was used to scan the entire arch area and find the maximum arch distance from the ground. The patients with arch distance $<1 \mathrm{~cm}$ were diagnosed with flatfoot. The results were consistent with that of a specialized physician among 20 subjects. A. O. Hamza et al. [87] proposed an optical footprint photographing method and correlative algorithms to provide a more specific evaluation of flatfoot. The patients' foot surface is kept on a transparent panel and shot by a camera from the opposite side. The captured image is binarized and divided into plantar contact and non-contact areas. Based on the ratio between the two areas, the foot deformity degree can be diagnosed and classified into 7 levels. In his work [88], Luis A. Navarro developed a flatfoot detection platform, composed of a webcam and 6 FSR sensors, to provide more comprehensive information about the foot structure. The webcam collected information on arch length and width, and the force sensors were used to obtain the pressure at discrete plantar regions. With these two kinds of information, the pressure distribution pattern across the whole plantar surface was then estimated.

TABLE III

\begin{tabular}{ccc}
\multicolumn{3}{c}{ PARAMETERS OF FLAT FOOT DIAGNOSIS } \\
\hline Parameters & Description & Flatfoot Patients \\
\hline Arch height & $\begin{array}{c}\text { Maximum arch distance } \\
\text { from the ground }\end{array}$ & $<1 \mathrm{~cm}$ \\
\hline $\begin{array}{c}\text { Chippaux- } \\
\text { Smirak index } \\
\text { (C-S index) }\end{array}$ & $\begin{array}{c}\text { Ratio between the minimum } \\
\text { arch width and the maximum } \\
\text { forefoot width }\end{array}$ & $>45 \%$ \\
\hline Barkhusen Index & $\begin{array}{c}\text { Ratio between contact and } \\
\text { non-contact area of plantar }\end{array}$ & $>2$ \\
\hline $\begin{array}{c}\text { CoP excursion } \\
\text { index (CPEI) }\end{array}$ & $\begin{array}{c}\text { Ratio between CoP deviation } \\
\text { toward the lateral foot } \\
\text { and foot width }\end{array}$ & $<14 \%$ \\
\hline $\begin{array}{c}\text { Ankle rotation } \\
\text { angle }\end{array}$ & $\begin{array}{c}\text { In both sagittal plane } \\
\text { and coronal plane }\end{array}$ & $\begin{array}{c}\text { Higher internal rotation, } \\
\text { higher plantarflexion angle } \\
\text { lower dorsiflexion angle }\end{array}$ \\
\hline $\begin{array}{c}\text { Stance phase } \\
\text { duration }\end{array}$ & / & $\begin{array}{c}\text { Lower in the early } \\
\text { stance phase }{ }^{\text {a) }}\end{array}$ \\
\hline \multicolumn{2}{c}{ a) The comparisons are relative to healthy people. }
\end{tabular}


Output results of this system were provided to specialists as an analysis tool.

Although several effective diagnostic platforms have been proposed, these techniques suffer from complex data collecting processes and large volumes. To address this issue, J. Y. Kim et al. [89] proposed an in-shoe sensing technology. The new system contains two force sensors and one angle sensor to measure gait-related signals; both types of sensors are based on piezoresistive techniques. The front and rear force sensors detect the strike or lifting of the toe and heel to identify gait phases, while the angle sensor provides information about ankle rotation angles. With a deep neural network model, the existence of flatfoot could be determined at an accuracy of $81.52 \%$. The in-shoe force-sensing method shows advantages in comfort and convenience; however, their accuracy is limited because only gait-related features are measured. In the future, it is expected to see portable flatfoot diagnosis equipment that integrates the measurement of both foot structure and gait features.

\section{E. Knee osteoarthritis}

Knee osteoarthritis (KOA) is a common chronic disease caused by knee joint damage and muscle weakness [92]. Patients with KOA have poor postural balance and special gait strategies. To monitor and analyze the motion abnormalities of KOA patients, critical indicators include plantar pressure distribution [94], CoP path [98], duration of gait phases, and knee adduction moment (KAM) [93, 99]. As listed in Table IV $[95,97,98]$, KOA patients tend to have a more dispersed plantar pressure distribution pattern, longer single support phase duration, smaller $\mathrm{CoP}$ path range, and higher KAM value during walking.

On the basis of medical studies, insoles with force-sensitive sensors have been designed for KOA patients. For example, I. Saito et al. [98] used the F-scan insole sensing system to detect the CoP path of KOA patients. In walking experiments, the percentage of the anteroposterior $\mathrm{CoP}$ path length to the foot length (\%Long) of patients was found to be significantly lower than normal people (52.4\% vs $64.7 \%$ ). This is because patients' limited knee movement angles will lead to a shorter anteroposterior displacement of CoP. M. M. Organero et al. [95] used an insole system with 8 FSR sensors to analyze the walking strategy of KOA patients. Through experiments with $14 \mathrm{KOA}$ patients and 14 healthy patients as the standard, the double support phase duration and forefoot pressure transfer mode (e.g., move speed and route) are found to have a close relationship with KOA. Each feature is able to identify KOA with an accuracy higher than $89 \%$, based on support vector machines (SVM) or decision tree algorithms.

In addition to plantar pressure and CoP path, KAM has also been proved as a critical indicator of KOA. A. Tiwari et al. [97] designed a system with a 6-axis goniometer and a 6-axis force/torque sensor to measure the KAM of KOA patients and healthy people during lower limb exercises. In the experiment, which consisted of stepping on an elliptical trainer, the mean peak KAM value of the patient group was found to be $47 \%$ higher. In another study [100], a wearable feedback system was proposed to reduce the patients' KAM value during walking. The system was composed of an insole FSR sensor located at the lateral side of the heel and a vibration motor at the ankle. When the detected pressure crossed a pre-determined threshold, the motor was activated to help patients produce a more medial weight-bearing strategy. Results showed that, through the feedback, reductions of $6.0 \%$ and $13.9 \%$ were achieved in the first and the second peak of KAM, respectively. In a study by A. M. Howell et al. [101], an insole with 12 FSRs was designed to estimate patients' KAM. The obtained plantar pressure was compared with KAM values which were simultaneously measured by motion capture cameras. Through a linear regression method (correlation index: 0.80), this KAM detection method achieved over $75 \%$ accuracy.

In the future, accurate measurements, from both plantar pressure and KAM, are desired for insole systems in order to give a more comprehensive and reliable diagnosis result for KOA patients.

\section{F. Elder falling event}

The prediction or warning of falling events, which may cause elder people life-threatening injuries, such as fractures and

TABLE IV

CRITICAL INDICATORS OF KOA PATIENTS

\begin{tabular}{ccc}
\hline Parameters & Patients with KOA & Health people \\
\hline $\begin{array}{c}\text { Forefoot pressure } \\
\text { transfer mode }\end{array}$ & $\begin{array}{c}\text { Dispersing between } \\
\text { the medial and the } \\
\text { center of the forefoot }\end{array}$ & $\begin{array}{c}\text { First load the central } \\
\text { part and then move to } \\
\text { the medial part }\end{array}$ \\
\hline $\begin{array}{c}\text { Single support phase } \\
\text { duration }\end{array}$ & - & Longer \\
\hline $\begin{array}{c}\text { Anteroposterior length } \\
\text { of CoP path }\end{array}$ & - & Higher \\
\hline $\begin{array}{c}\text { Transverse width } \\
\text { of CoP path }\end{array}$ & - & Higher \\
\hline Peak value of KAM & Higher & - \\
\hline
\end{tabular}

visceral rupture, is strongly expected in elderly support services [102-105]. The concerned gait information for falling detection include CoP displacement, total CoP displacement, step length, and gait velocity $[106,107]$. Note that, the CoP refers to the pressure center of a single foot at the walking or standing state, while $\mathrm{CoP}$ emphasizes the pressure center of both feet during the standing posture. For the detection of Falling, parameters including ground reaction force deviation; gait phase and duration are often detected for the measurement of deviation of CoP.

Early-stage studies observed the gait of elder people in laboratory environments using fixed equipment such as depth cameras. For example, A. Dubois et al. [108] developed a system based on an RGB-D camera to assess fall risk. Based on image processing algorithms, the human center of mass trajectory was estimated, which was then utilized to obtain gait parameters, such as step length, step duration, and gait velocity. The estimation error for the three parameters was verified by a commercial altimetric carpet (GAITRite) to be less than 6.6\%. The depth camera-based system provides reliable sensing ability; however, it is cumbersome and extremely restricted by the venue.

To improve the convenience of the monitoring process, $\mathrm{H}$. A. Ghaida et al. [109] introduced a wearable insole with three FSR sensors to monitor people's CoP during the standing posture. 
The experimental results showed a distinct correlation between the developed insole and F-Scan insole system (Tekscan, USA), which is the most generally used insole system by podiatrists [109]. The average root mean square (RMS) error of CoPT measurement was $3 \mathrm{~mm}$ in the mediolateral direction and $2 \mathrm{~mm}$ in the anteroposterior direction. D. Chen et al. [112] proposed a smart insole with a 96 piezoresistive sensor array and an IMU to report elderlies' falling types. In their experiments, parameters include max ground reaction force difference, foot contact pitch, double-support phase duration, and pressure threshold-crossing point number are used to identify fall risks due to their close relationship with body balance changes. With an SVM classifier, two types of falling events (i.e., slip and trip) could be recognized with an accuracy of $98.1 \%$. L. Wang et al. [110] developed a smart insole constructed with a 3-axis accelerometer and a 3-axis gyroscope, to recognize falling events in elderlies' daily activities. A one-dimension convolutional neural network model was used to process the raw sensor data, achieving an accuracy of $98.61 \%$. Compared with the traditional fall hazard identification method which relies on cameras, the insole-based systems provide more convenience and lower costs.

Apart from identifying falling events during walking, insolebased systems have also been utilized for falling risks prediction. For instance, J. C. Ayena et al. [111] conducted a One-Leg Standing test for falling risk assessment based on 4 FSR sensors placed at heel and toes areas. The CoP line changes were calculated during experiments and compared with the participants' assessment of falling risk based on the Likert scale. Results showed that the swing of $\mathrm{CoP}$ is an effective indicator for elderly falling risk evaluation. Although various smart insole sensors have been proposed to detect and prevent accidental falls, there are still several problems preventing them from practical applications. The first problem is comfort. The insole sensor itself can now be thin and flexible; however, the circuit board with battery and wireless module still takes up a large volume, causing users' discomfort. Second, the energy consumption problem cannot be ignored. Insole sensors and data transmission need a constant energy supply during longterm daily monitoring, however, current battery technology can hardly meet this demand. With the development of flexible circuits and advanced energy management, those problems are expected to be solved in the near future, so that the insole-based systems can be used in fall detection on a large scale.

These six representative chronic diseases can be classified into neurologic, orthopedic and musculoskeletal types. Their specified illustrations of jeopardy and cause are shown in Fig.5. As a result of the fruitful outcomes and precise results of former research, clinical features (based on plantar pressure) of each disease have been extracted. Additionally, we have demonstrated the development process and parameters of interest of the corresponding medical insole systems for the use of medical professionals.

\section{CONCLUSION AND OUTLOOK}

\section{A. Challenges}

1) Black-box issue in disease diagnosis

To provide disease detection at a high level of accuracy by machine learning algorithms, a massive database is strongly expected and demanded. Although currently no such a database is available, researchers from diverse institutions have established small-scale databases for specific disease diagnosis, and some fruitful results have been reported as discussed in previous sections. Nevertheless, the outputted results from machine learning models cannot be directly utilized for disease analysis yet. One of the key reasons is that the determination process of AI algorithms hasn't been fully understood by human beings, i.e., the so called black-box issue.

The black-box issue hinders the development of machine learning supported disease analysis, especially in the application scenarios of the IoHT, in which diagnosis data are sent to remote medical staff without explanation as to how the results are generated. Unlike areas such as automatic driving, in which results obtained through machine learning can be used to make decisions directly (e.g., speed up or U-turn), medical data needs to be fully understood before giving further treatments. Therefore, current machine learning based techniques are majorly used as assistive tools, thus losing their main power in addressing non-linear complex issues like diagnosing diseases through gait features.

To summarize, present dilemma consists of the black-box issue, the lack of a large database and hindered development of IoHT.

\section{2) Intrinsic features of gait parameters}

In addition to the challenge of establishing a giant database with ample data as mentioned above, another current dilemma involves issues associated with gait parameters. Seeing that gait parameters are a subset of biological data, algorithm training runs into the danger of becoming biased.

This phenomenon can be attributed to intrinsic features of gait parameters, as duplicate or near-duplicate samples account for the highest proportion in biological data [122]. Since gait parameters are generated from similar daily activities, the duplicate phenomenon is more obvious. Changes of some parameters are not obviously reflected in a short period. When constructing a database for machine learning with gathered parameters, duplicate samples appear frequently in training and testing datasets. Hence, the miniscule changes of some parameters, which arise from chronic diseases, may be neglected by the training algorithms.

In essence, although algorithms can train machine learning models effectively, the influence of neglected features cannot be reflected properly. Thence, the algorithms might be unintentionally biased based on the features extracted, and the accuracy of evaluation for rehabilitation and/or diagnosis will decrease [123].

\section{B. Outlook and Conclusion}

This article demonstrates the clinical features and pathogenic mechanisms of six neurologic and orthopedic chronic diseases. The development of their corresponding insole systems for diagnosis and rehabilitation are reviewed as well.

In the main part, five insole sensing techniques and their representative current products (piezoelectric, resistive, piezoresistive, capacitive and temperature-humidity) are introduced. Their merits and drawbacks can be used as a guideline for medical staff. Next, clinical gait parameters of six specific diseases and their insole systems-based diagnosis and 
rehabilitation methods are reviewed in detail. These parts can provide considerable guidance for medical professionals while conducting gait and posture-based research of diseases.

In the foreseeable future, we think that gait monitoring in disease analysis will progress towards the following trends.

1) Predict Patient's Performance under Different Scenarios

In rehabilitation trainings, patients are requested to perform different tasks, in order to obtain desired information for designing proper assistive products that will enhance their performances in daily activities. However, the training activities cannot cover all life scenes. Therefore, methods for using limited gait data to predict patients' behaviors in most of life scenes, if not all, will be greatly helpful. For example, through the gait data of walking in a solid flat road, algorithms can predict corresponding performance on rugged roads. Such algorithms have been developed for applications like Designing Deep Reinforcement Learning Systems for Musculoskeletal Modeling and Locomotion Analysis Using Wearable Sensor Feedback; Phase-Functioned Neural Networks for Character Control; Local Motion Phases for Learning Multi-Contact Character Movements. These scenarios indicate the potential of developing desired algorithms for gait data.

2) Establishing Relationships Between Machine Learning Models and Musculoskeletal Nervous System

As discussed in the previous section, machine learning methods are treated as black boxes. Although they can provide high analysis accuracy, the lack of intermediate procedures raise medical staff's worries. As gait is the comprehensive result of neuros and orthopedic organs, gait information can potentially bridge the gap between the black box generated results and the results obtained from the musculoskeletal nervous system, thus providing doctors reasonable explanations. 3) Human Body Digital Twin

Digital twin technology, refers to the replication of an object in a virtual reality setting. By integrating diverse sensors into the object and employing comprehensive algorithms to manipulate the historical and real-time data provided by the sensors, this technology proved to be feasible in studying the object's current working status and predicting its change under different conditions. Construction of a human body's DT model for disease analysis is treated as an impossible task due to the lack of large volume datasets retrieved from conventional body motion monitoring technologies. Nevertheless, insole systems can track most humans' activities during the daytime, indicating the possibility of providing essential data for constructing human DT models. Hence, it is worthy to note that multisensory fusion technologies with other kinds of wearables would strongly benefit the creation of human body DT models.

\section{REFERENCES}

[1] Australian Institute of Health and Welfare. Chronic Diseases. (2016). Available from: http://www.aihw.gov.au/chronic-diseases/

[2] Yuehong Y I N, Zeng Y, Chen X, et al. The internet of things in healthcare: An overview[J]. Journal of Industrial Information Integration, 2016, 1: 313.

[3] L. K. Vognild, T. M. Burkow and L. F. Luque, "The MyHealthService approach for chronic disease management based on free open source software and low cost components," 2009 Annual International Conference of the IEEE Engineering in Medicine and Biology Society, 2009, pp. 1234-1237, doi: 10.1109/IEMBS.2009.5333475.
[4] Bai, B, Nazir, S, Bai, Y, Anees, A. Security and provenance for Internet of Health Things: A systematic literature review. J Softw Evol Proc. 2021; 33: e2335. https://doi.org/10.1002/smr.2335.

[5] Feng L, Jing-Long H, Ji Q, et al. Research and application progress of intelligent wearable devices[J]. Chinese Journal of Analytical Chemistry, 2021, 49(2): 159-171.

[6] H. Jin, Y. Luo, P. Li and J. Mathew, "A Review of Secure and PrivacyPreserving Medical Data Sharing," in IEEE Access, vol. 7, pp. 6165661669, 2019, doi: 10.1109/ACCESS.2019.2916503.

[7] Logroscino G. The role of early life environmental risk factors in Parkinson disease: what is the evidence? [J]. Environmental health perspectives, 2005, 113(9): 1234-1238.

[8] F. Moissenet, C. Bélaise, E. Piche, B. Michaud, and M. Begon, "An optimization method tracking EMG, ground reactions forces and marker trajectories for musculo-tendon forces estimation in equinus gait," Front. Neurorob., vol. 13, pp. 48, 2019.

[8] F. Moissenet, C. Bélaise, E. Piche, B. Michaud, and M. Begon, "An optimization method tracking EMG, ground reactions forces and marker trajectories for musculo-tendon forces estimation in equinus gait," Front. Neurorob., vol. 13, pp. 48, 2019.

[9] H. A. Ghaida, S. Mottet and J. Goujon, "A real time study of the human equilibrium using an instrumented insole with 3 pressure sensors," 2014 36th Annual International Conference of the IEEE Engineering in Medicine and Biology Society, Chicago, IL, USA, 2014, pp. 4968-4971.

[10] Darak B S, Hambarde S M. A review of techniques for extraction of cardiac artifacts in surface EMG signals and results for simulation of ECGEMG mixture signal[C]//2015 International Conference on Pervasive Computing (ICPC). IEEE, 2015: 1-5.

[11] Reaz M B I, Hussain M S, Mohd-Yasin F. Techniques of EMG signal analysis: detection, processing, classification and applications[J]. Biological procedures online, 2006, 8(1): 11-35.

[12] W. Yao, C. Chu and Z. Li, "The use of RFID in healthcare: Benefits and barriers," 2010 IEEE International Conference on RFID-Technology and Applications, 2010, pp. 128-134, doi: 10.1109/RFID-TA.2010.5529874.

[13] H. A. Nahas and J. S. Deogun, "Radio frequency identification applications in smart hospitals," in Proceedings of the 20th IEEE International Symposium on Computer-Based Medical Systems, 2007, pp. 337-342.

[14] S. J. M. Bamberg, P. LaStayo, L. Dibble, J. Musselman, and S. K. D. Raghavendra, "Development of a quantitative in-shoe measurement system for assessing balance: sixteen-sensor insoles," in Annu. Int. Conf. IEEE Eng. Med. Biol. Proc., New York, NY, USA, 2006, pp. 6041-6044.

[15] Tekscan Inc., "Pressure mapping, force measurement \& tactile sensors", [Online]. Available: https://www.tekscan.com/productssolutions/systems/f-scan-system, Accessed on: Aug. 6, 2020.

[16] C. Gerlach, D. Krumm, M. Illing, J. Lange, O. Kanoun, S. Odenwald, and A. Hübler, "Printed MWCNT-PDMS-composite pressure sensor system for plantar pressure monitoring in ulcer prevention," IEEE Sens. J., vol. 15 , no. 7, pp. 3647-3656, 2015

[17] A. Amemiya, H. Noguchi, M. Oe, H. Sanada, and T. Mori, "Establishment of a measurement method for in-shoe pressure and shear stress in specific regions for diabetic ulcer prevention, in Proc. Annu.Int. Conf. IEEE Eng. Med. Biol. Soc., Orlando, FL, USA, 2016, pp.2291-2294..

[18] F. Melakessou, W. Bieck, Q. Lallemant, G. Palmirotta, and B. Anti,"Presentation of a new sensor enabling reliable real time foot plantar pressure distribution retrieval," in Lect. Notes Inst. Comput. Sci. Soc.Informatics Telecommun. Eng., Vienna, Auatria, 2017, pp. 217-224.

[19] R. F. Lind, L. J. Love, J. C. Rowe, and F. G. Pin, "Multi-axis foot reaction force/torque sensor for biomedical applications," in IEEE/RSJ Int. Conf. Intelligent Rob. Syst., St Louis, MO, 2009, pp. 2575-2579.

[20] Y. A. Mustufa, J. Barton, B. O'Flynn, R. Davies, P. McCullagh, and H.Zheng, "Design of a smart insole for ambulatory assessment of gait," in IEEE Int. Conf. Wearable Implant. Body Sens. Netw., Cambridge, MA, USA, 2015, pp. 1-5.

[21] B. J. Braun, E. Bushuven, R. Hell, N. T. Veith, J. Buschbaum, J. H. Holstein, and T. Pohlemann, "A novel tool for continuous fracture aftercare-clinical feasibility and first results of a new telemetric gait analysis insole, Injury, vol. 47, no. 2, pp. 490-494, 2016.

[22] A. Putti, G. Arnold, L. Cochrane, and R. Abboud, "The Pedar® in-shoe system: Repeatability and normal pressure values," Gait Posture, vol. 25, no. 3, pp. 401-405, 2007 
[23] C. Giacomozzi, and L. Uccioli,"Learning from experience: A simple effective protocol to test footwear prescriptions for the Diabetic foot by using the Pedar system," J. Biomed. Sci. Eng., vol. 6, pp. 45-57, 2013.

[24] B. Mariani, H. Rouhani, X. Crevoisier, and K. Aminian, "Quantitative estimation of foot-flat and stance phase of gait using foot-worn inertial sensors," Gait Posture, vol. 37, no. 2, pp. 229-234, 2013.

[25] S. Kärki, J. Lekkala, H. Kuokkanen, and J. Halttunen, "Development of a piezoelectric polymer film sensor for plantar normal and shear stress measurements," Sens. Actuators, A, vol. 154, no. 1, pp. 57-64, 2009.

[26] I. Lourenço and J. Costa, "In-shoe Sensor System with an Embedded User Interface and Wearable Leg Unit," 2019 IEEE 6th Portuguese Meeting on Bioengineering (ENBENG), 2019, pp. 1-4, doi: 10.1109/ENBENG.2019.8692453.

[27] C. Jordan and R. Bartlett, "Pressure distribution and perceived comfort in casual footwear," Gait Posture, vol. 3, no. 4, pp. 215-220,1995.

[28] A. J. Boulton, R. P. Betts, C. I. Franks, P. G. Newrick, J. D.Ward, and T. Duckworth, "Abnormalities of foot pressure in early diabetic neuropathy," Diabetes Med., vol. 4, no. 3, pp. 225-228, May/Jun. 1987.

[29] D. Norton, "Calculating the risk: Reflections on the norton scale," Decubitus, vol. 2, no. 3, pp. 24-31, Aug. 1989.

[30] P. A. Keller, S. P. Sinkovic, and S. J. Miles, "Skin dryness: A major factor in reducing incontinence dermatitis," Ostomy Wound Manage, vol. 30, pp. 60-64, Sep./Oct. 1990.

[31] P. Brand, "Diabetes mellitus: Theory and practice," in The Diabetic Foot, M. R. Ellenberg, H. Editors, Ed. New Hyde Park, NY, USA: Med.Examination Publishing, 1983, pp. 829-849.

[32] D. G. Armstrong, P. J. Liswood, and W. F. Todd, "Contralateral limb during total contact casting-A dynamic pressure and thermometric analysis," J. Amer. Podiatric Med. Assoc., vol. 85, no. 12, pp. 733-737, Dec. 1995.

[33] A. M. Cristiani, G. M. Bertolotti, E. Marenzi and S. Ramat, "An Instrumented Insole for Long Term Monitoring Movement, Comfort, and Ergonomics," in IEEE Sensors Journal, vol. 14, no. 5, pp. 1564-1572, May 2014, doi: 10.1109/JSEN.2014.2299063.

[34] T. Bernard, C. D'Elia, R. Kabadi and N. Wong, "An early detection system for foot ulceration in diabetic patients," 2009 IEEE 35th Annual Northeast Bioengineering Conference, 2009, pp. 1-2, doi: 10.1109/NEBC.2009.4967797.

[35] W. Shue, N. Paudyal, M. Rabiah, D. E. Dow, M. Ergezer and M. Yun, "A Thermally Regulated Footwear \& Alerting System," 2019 IEEE 10th Annual Ubiquitous Computing, Electronics \& Mobile Communication Conference (UEMCON), 2019, pp. 0179-0182, doi: 10.1109/UEMCON47517.2019.8992977.

[36] J. Jankovic, "Parkinson disease: Clinical features and diagnosis," J.Neurol. Neurosurg. Psychiatry, vol. 79, pp. 368-376, 2008.

[37] M. E. Morris, F. Huxham, J. McGinley, K. Dodd, and R. Iansek, "The biomechanics and motor control of gait in Parkinson disease," Clin.Biomechan., vol. 16, no. 6, pp.459-470,2001.

[38] P. Damier, "Evidence for the use of levodopa-carbidopa-entacapone (STALEVO) to improve motor fluctuations in Parkinson disease,"Eur. Neurol. Rev., vol. 3, no. 2, pp.37-40, 2008.

[39] T. Morris, C. Cho, et al., "Clinical assessment of freezing of gait in Parkinson's disease from computer-generated animation, "Gait \& posture, 38(2) (2013): 326-329.

[40] Rochester L, Nieuwboer A, Baker K, Hetherington V , Willems A.M , Chavret F, Kwakkel G, Wegen E, Lim I, Jones D. The attentional cost of external rhythmical cues and their impact on gait in Parkinson disease: effect of cue modality and task complexity. J Neural Transm 2007; 114: 1243-1248

[41] K. Grandez, P. Bustamante, G. Solas, I. Gurutzeaga and A. García-Alonso, "Wearable wireless sensor for the gait monitorization of Parkinsonian patients," 2009 16th IEEE International Conference on Electronics, Circuits and Systems - (ICECS 2009), 2009, pp. 215-218, doi: 10.1109/ICECS.2009.5410974.

[42]S. Corbellini, C. Ramella, C. Fallauto, M. Pirola, S. Stassi and G. Canavese, "Low-cost wearable measurement system for continuous real-time pedobarography," 2015 IEEE International Symposium on Medical Measurements and Applications (MeMeA) Proceedings, 2015, pp. 639644, doi: 10.1109/MeMeA.2015.7145281.
[43] D. Rosenbaum end H.P. Becker, "Planar pressure distribution measurements: technical backgorund and clinical applicaitons," Foot Ankle Surg.,vol. 3, pp. 1-14, 1997.

[44] L. Wafai et al., "Asymmetry detection during pathological gait using a plantar pressure sensing system," Proc. IEEE GCC Conf. Exhib. pp. 182187,2013

[45] R. Hua and Y. Wang, "Monitoring Insole (MONI): A Low Power Solution Toward Daily Gait Monitoring and Analysis," in IEEE Sensors Journal, vol. 19, no. 15, pp. 6410-6420, 1 Aug.1, 2019, doi: 10.1109/JSEN.2019.2910105

[46] J. Barth et al., "Biometric and mobile gait analysis for early diagnosis and therapy monitoring in Parkinson disease," in Proc. IEEE 31st Annu. Int. Conf. Eng. Med. Biol. Soc. (EMBC), Boston, MA, USA,Aug. 2011, pp. $868-871$.

[47] X.Wu, X.Chen, Y.Duan, S.Xu, N.Cheng and N.An, "A study on gait-based Parkinson's disease detection using a force sensitive platform," 2017 IEEE International Conference on Bioinformatics and Biomedicine (BIBM), 2017, pp. 2330-2332, doi: 10.1109/BIBM.2017.8218048.

[48] E. Jovanov, E.Wang, L.Verhagen, M.Fredrickson and R.Fratangelo, "deFOG-A real time system for detection and unfreezing of gait of Parkinsons patients," 2009 Annual International Conference of the IEEE Engineering in Medicine and Biology Society, 2009, pp. 5151-5154, doi: 10.1109/IEMBS.2009.5334257.

[49] M. Bachlin et al., "Wearable Assistant for Parkinson's Disease Patients With the Freezing of Gait Symptom," in IEEE Transactions on Information Technology in Biomedicine, vol. 14, no. 2, pp. 436-446, March 2010, doi: 10.1109/TITB.2009.2036165.

[50] C. Pongmala, A. Suputtitada and M. Sriyuthsak, "The study of cueing devices by using visual, auditory and somatosensory stimuli for improving gait in Parkinson patients," 2010 International Conference on Bioinformatics and Biomedical Technology, 2010, pp. 185-189, doi: 10.1109/ICBBT.2010.5478983.

[51] Stockl K, Vanderplas A, Tafesse E et al. Costs of lower-extremity ulcers among patients with diabetes. Diabetes Care. 2004;27(9):2129-34

[52] R. E. Morley, E. 1. Richter, 1. W. Klaesner, K. S. Maluf, M. 1. Mueller, "In-Shoe Multisensory Data Acquisition System," IEEE Trans. Biom. vol. 48, no. 7, July 2001.

[53] S. Urry, "Plantar pressure-measurement sensors," Measurement Science and Technology, vol. 10, pp. R16-R32, 1999.

[54] Y. Wahab, A. Zayegh, R. K. Begg and R. Veljanovski, "Design of MEMS biomedical pressure sensor for gait analysis," 2008 IEEE International Conference on Semiconductor Electronics, 2008, pp. 166-169, doi: 10.1109/SMELEC.2008.4770300.

[55] A. Amemiya et al., "Relationship between elevated plantar pressure of toes and forefoot and gait features in diabetic patients," 2013 35th Annual International Conference of the IEEE Engineering in Medicine and Biology Society (EMBC), 2013, pp. 4633-4636, doi: 10.1109/EMBC.2013.6610580.

[56] M. Nouman, S. Chatpun, T. Prachgosin and W. Leelasamran, "Plantar pressure distribution in non-obese, overweight and obese subjects with diabetic neuropathy while walking," 2018 11th Biomedical Engineering International Conference (BMEiCON), 2018, pp. 1-4, doi: 10.1109/BMEiCON.2018.8609969.

[57] P. Aqueveque, R. Osorio, F. Pastene, F. Saavedra and E. Pino, "Capacitive Sensors Array for Plantar Pressure Measurement Insole fabricated with Flexible PCB," 2018 40th Annual International Conference of the IEEE Engineering in Medicine and Biology Society (EMBC), 2018, pp. 43934396, doi: 10.1109/EMBC.2018.8513383.

[58] P. E. Crago, H. J. Chizeck, M. R. Neuman, and F. T. Hambrecht, "Sensors for use with functional neuromuscular stimulation" IEEE Transactions on Biomedical Engineering, vol. BME-33, pp 256-268, 1986

[59] Jan Rueterbories, Erika G. Spaich, Birgit Larsen, Ole K. Andersen,"Methods for gait event detection and analysis in ambulatory systems" Medical Engineering \& Physics, vol. 32, no. 6, pp. 545-552, 2010.

[60] M. A. BENCHEIKH and S. BOUKHENOUS, "A low Cost Smart Insole for Diabetic Foot Prevention," 2018 International Conference on Applied Smart Systems (ICASS), 2018, pp. 1-4, doi: 10.1109/ICASS.2018.8651973.

[61] M.Attari, and S.Boukhenous, "A Tactile Sensors Array for Biomedical Applications, "Proceeding of 5th International Multi-Conference on 
Systems, Signals and Devices, IEEESSD'08, ISBN: 978-1-4244-2206-7, Amman, Jordanie, Juillet 20-23, 2008.

[62]P. Ripka, and A. Tipek, "Modern Sensors Handbook," ISTE Ltd, UK, 2007, $536 \mathrm{p}$.

[63] International Diabetes Federation. Diabetes atlas 5th edition, 2011. Available from http://www.idf.org/media-events/pressreleases/2011/Diabetes-atlas-5th-edition, Accessed 17 January 2013

[64] M.J. Hessert, M. Vyas, J. Leach, K. Hu, L. A. Lipsitz, and V. Novak, "Foot pressure distribution during walking in young and old adults, "BMC Geriatr., vol. 5, p. 8, May 2005.

[65] K. Alexiadou and J. Doupis, "Management of diabetic foot ulcers," Diabetes Ther., vol. 3, no. 1, Dec. 2012.

[66] A.Albathi, A.AlQahtani, H.Alshagawi, S.Almoammer, E.Al-Fakih and L. ElSahmarany, "DESIGN OF A SMART IN-SOLE TO MODEl AND CONTROL THE PRESSURE UNDER DIABETIC PATIENTS FEET," 2019 8th International Conference on Modeling Simulation and Applied Optimization (ICMSAO), 2019, pp. 1-6, doi: 10.1109/ICMSAO.2019.8880355.

[67] A. N. Dewanjee, Q. D. Hossain and A. Muhury, "Quantitative Deviation of Spatial Parameters of Gait in Parkinson Disease," 2019 International Conference on Wireless Communications Signal Processing and Networking (WiSPNET), 2019, pp. 304-309, doi: 10.1109/WiSPNET45539.2019.9032870.

[68] M. Yoneyama, Y. Kurihara, K. Watanabe, and H. Mitoma, "Accelerometry-Based Gait Analysis and Its Application to Parkinson's Disease Assessment-Part 2: A New Measure for Quantifying Walking Behavior," in IEEE Transactions on Neural Systems and Rehabilitation Engineering, vol. 21, no. 6, pp. 999-1005, Nov. 2013, doi: 10.1109/TNSRE.2013.2268251.

[69] Novak P, Novak V. Effect of step-synchronized vibration stimulation of soles on gait in Parkinson disease: a pilot study. J NeuroEng Rehab 2006; 3:9.

[70] Lim E , Tan T.M, Seet R. LASER-Assisted Device(LAD) for start hesitation and freezing in Parkinson disease. Case Rep Clin Pract Rev 2006; 7: 92-95.

[71] Armstrong DG, Peters EJ, Athanasiou KA, Lavery LA. Is there a critical level of plantar foot pressure to identify patients at risk for neuropathic foot ulceration? J Foot Ankle Surg 1998;37:303-307pmid:9710782

[72] Edmonds ME, Blundell MP, Morris ME et al. Improved survival of the diabetic foot: the role of a specialized foot clinic. Q J Med. 1986;60(232):763-71

[73] C. Warlow, J. van Gijn, M. Dennis, J. Wardlaw, J. Bamford, G. Hankey, P. Sandercock, G. Rinkel, P. Langhorne, C. Sudlow and P. Rothwell, Stroke - Practical Management, Oxford: Blackwell Publishing, 2008.

[74] P. Langhorne, F. Coupar and A. Pollock, „Motor recovery after stroke," Lancet Neurol, vol. 8, pp. 741-54, 2009.

[75] WHO Repot, "Cardiovascular diseases: Facts about cardiovascular diseases", 2009.

[76] G. Ferraresi, M. Manca, A. Leardini, et al, "Gait pattern classification in hemiplegic patients with equinus deformity," Gait \& Posture, vol. 33, pp. S14-S15, 2011.

[77] A. H. Abdul-Razak, A. Zayegh, R. K. Begg, Y. Wahab, "Foot Plantar Pressure Measurement System: A Review," Sensors, vol. 12, no. 7, pp. 9884-9912, 2012.

[78] M. Munoz-Organero, J. Parker, L. Powell, and S. Mawson, "Assessing walking strategies using insole pressure sensors for stroke survivors," Sensors, vol. 16, no. 10, pp. 1-18, Oct. 2016. [Online]. Available: http://www.mdpi.com/1424-8220/16/10/1631

[79] P. L. Meyer, G. D. Fulk and E. S. Sazonov, "Automatic Detection of Temporal Gait Parameters in Poststroke Individuals," in IEEE Transactions on Information Technology in Biomedicine, vol. 15, no. 4, pp. 594-601, July 2011, doi: 10.1109/TITB.2011.2112773.

[80] A. M. Howell, T. Kobayashi, T. R. Chou, W. Daly, M. Orendurff, and S. J. M. Bamberg, "A laboratory insole for analysis of sensor placement to determine ground reaction force and ankle moment in patients with stroke," in Proc. 34th Annu. Int. Conf. IEEE Eng. Med. Biol. Soc. (EMBC), San Diego, CA, USA, Aug. 2012, pp. 6394-6397.

[81] L. Qin, H. Ma and W. Liao, "Insole plantar pressure systems in the gait analysis of post-stroke rehabilitation," 2015 IEEE International Conference on Information and Automation, 2015, pp. 1784-1789, doi: 10.1109/ICInfA.2015.7279576.
[82] M. M. Organero, J. Parker, L. Powell, R. Davies and S. Mawson, "Sensor Optimization in Smart Insoles for Post-Stroke Gait Asymmetries Using Total Variation and L1 Distances," in IEEE Sensors Journal, vol. 17, no. 10, pp. 3142-3151, 15 May15, 2017, doi: 10.1109/JSEN.2017.2686641.

[83] Cai JinFang, Department of Orthopaedics and trauma, General Hospital of Jinan Military Region, 2013, "Chinese Journal of Orthopaedics, issue 6"

[84] Han Yankun; Huo Hongfeng Summary of papers of the 11th National Sports Science Conference

[85] Phan Cong-Bo; Lee Kyoung Min; Kwon Soon-Sun; Koo Seungbum Journal of Biomechanics

[86] AO Hamza, HK Ahmed, MO Khider, "A New Noninvasive Flatfoot Detector", 2015

[87] Arnesh Sen, Kaustav Sen, Jayoti Das, "In - House Early Detection of Flat Foot and High Ankle Foot Using Image Processing", July 6-8, 2019

[88] L. A. Navarro et al., "Opto-electronic system for detection of flat foot by using estimation techniques: Study and approach of design," 2010 Annual International Conference of the IEEE Engineering in Medicine and Biology, 2010, pp. 5768-5771, doi: 10.1109/IEMBS.2010.5627842.

[89] J. -Y. Kim, J. Y. Hwang, E. Park, H. -U. Nam and S. Cheon, "Flat-Feet Prediction Based on a Designed Wearable Sensing Shoe and a PCA-Based Deep Neural Network Model," in IEEE Access, vol. 8, pp. 199070-199080, 2020, doi: 10.1109/ACCESS.2020.3033826.

[90] Diagnosis and Treatment of Adult Flatfoot

[91] Changes in gait associated with acute stage II posterior tibial tendon dysfunction

[92] Tyler Lawaon, Adam Morrison, stephen Blaxland, Matthew wenman, Curtis G, schmidt, and Michael A. Hunt, "Laboratory- based measurement of standing balance in individuals with knee osteoarthritis: A systematic review," Clinical Biomechanics, Vol. 30, pp. 49-54, 2015.

[93] Z. He, T. Liu and J. Yi, "A Wearable Sensing and Training System: Towards Gait Rehabilitation for Elderly Patients With Knee Osteoarthritis," in IEEE Sensors Journal, vol. 19, no. 14, pp. 5936-5945, 15 July15, 2019, doi: 10.1109/JSEN.2019.2908417.

[94] M. Yamada and K. Nagamune, "A Development of Measurement System for Foot Pressure by Using Optical Force Sensors," 2018 World Automation Congress (WAC), 2018, pp. 1-5, doi: 10.23919/WAC.2018.8430401.

[95] M. M. Organero, C. Littlewood, J. Parker, L. Powell, C. Grindell and S. Mawson, "Identification of Walking Strategies of People With Osteoarthritis of the Knee Using Insole Pressure Sensors," in IEEE Sensors Journal, vol. 17, no. 12, pp. 3909-3920, 15 June15, 2017, doi: 10.1109/JSEN.2017.2696303.

[96] M. Yamada and K. Nagamune, "A Development of Measurement System for Foot Pressure by Using Optical Force Sensors," 2018 World Automation Congress (WAC), 2018, pp. 1-5, doi: 10.23919/WAC.2018.8430401.

[97] S. H. Kang, S. J. Lee, J. M. Press and L. -Q. Zhang, "Real-Time ThreeDimensional Knee Moment Estimation in Knee Osteoarthritis: Toward Biodynamic Knee Osteoarthritis Evaluation and Training," in IEEE Transactions on Neural Systems and Rehabilitation Engineering, vol. 27, no. 6, pp. 1263-1272, June 2019, doi: 10.1109/TNSRE.2019.2915812.

[98] I. Saito et al., "Foot pressure pattern and its correlation with knee range of motion limitations for individuals with medial knee osteoarthritis," Arch. Phys. Med. Rehabil., vol. 94, no. 12, pp. 2502-2508, 2013.

[99] Zexia He, Yang Shen, Tao Liu, Jingang Yi and J. P. Ferreira, "A gait retraining feedback system based on wearable sensors," 2017 IEEE International Conference on Advanced Intelligent Mechatronics (AIM), 2017, pp. 1029-1034, doi: 10.1109/AIM.2017.8014154.

[100] Erhart-Hledik J C, Asay J L, Clancy C, et al. Effects of active feedback gait retraining to produce a medial weight transfer at the foot in subjects with symptomatic medial knee osteoarthritis[J]. Journal of Orthopaedic Research, 2017, 35(10): 2251-2259.

[101] Howell A M, Kobayashi T, Hayes H A, et al. Kinetic gait analysis using a low-cost insole[J]. IEEE Transactions on Biomedical Engineering, 2013, 60(12): 3284-3290.

[102] S. S. Rao, "Prevention of falls in older patients," Amer. Family Phys., vol. 72, no. 1, pp. 81-88, Jul. 2005.

[103] T. Masud and R. O. Morris, "Epidemiology of falls," Age Ageing, vol. 30, no. S4, pp. 3-7, Nov. 2001. 
[104] J. H. Downton and K. Andrews, "Prevalence, characteristics and factors associated with falls among the elderly living at home," Aging Clin. Experim. Res., vol. 3, no. 3, pp. 219-228, Sep. 1991.

[105] M. E. Tinetti, M. Speechley, and S. F. Ginter, "Risk factors for falls among elderly persons living in the community," New England J. Med., vol. 319, no. 26, pp. 1701-1707, Dec. 1988.

[106] D. J. Hewson, N. K. Singh, H. Snoussi, \& J. Duchêne. Classification of elderly as fallers and non-fallers using Centre of Pressure velocity. Annual International Conference of the IEEE Engineering in Medicine and Biology Society (EMBS), 3678-3681, 2010.

[107] A. Miraoui, H. Snoussi, J. Duchêne, \& N. Azzaoui, (2010). On the detection of elderly equilibrium degradation using multivariate-EMD. IEEE Globecom 2010 Workshop on Advances in Communications and Networks, 2010, pp. 2049-2053.

[108] A. Dubois and F. Charpillet, "A gait analysis method based on a depth camera for fall prevention," 2014 36th Annual International Conference of the IEEE Engineering in Medicine and Biology Society, Chicago, IL, USA, 2014, pp. 4515-4518.

[109] S. A. Bus, J. S. Ulbrecht, and P. R. Cavanagh. Pressure relief and load redistribution by custom-made insoles. Clinical Biomechanics, 19, 629638, 2004.

[115] J. Wu, Y. Yang, X. Cheng, H. Zuo and Z. Cheng, "The Development of Digital Twin Technology Review," 2020 Chinese Automation Congress (CAC), 2020, pp. 4901-4906, doi: 10.1109/CAC51589.2020.9327756.

[116] M. F. Domingues et al., "Insole Optical Fiber Sensor Architecture for Remote Gait Analysis-An e-Health Solution," IEEE Internet. Things. vol. 6, no. 1, pp. 207-214, Feb. 2019.

[117] S. Siyang, T. Nilpanapan, and T. Kerdcharoen, "Development of IoT Based Data Shoes for Daily Life Step Count," 2018 IEEE 7th Global Conference on Consumer Electronics (GCCE), 2018: IEEE, pp. 524-525.

[118] P. S. Malvade, A. K. Joshi, and S. P. Madhe, "IoT based monitoring of foot pressure using FSR sensor," 2017 International Conference on Communication and Signal Processing (ICCSP), 2017: IEEE, pp. 06350639.

[119] O. Moore, C. Peretz, N. Giladi, "Freezing of gait affects quality of life of peoples with Parkinson's disease beyond its relationships with mobility and gait," Movement Disorders 2008;22:2192-95.

[120] Parkinson's Foundation Statistics. [Online]. Available: http://parkinson.org/Understanding-Parkinsons/CausesandStatistics/Statistics, 2018.

[121] A. Nieuwboer, W. De Weerdt, R. Dom, and E. Lesaffre, "A frequency and correlation analysis of motor deficits in Parkinson patients," Disabil. Rehabil., vol. 20, no. 4, pp. 142-150, 1998.

[122] Chen, Q.; Britto, R.; Erill, I.; Jeffery, C.J.; Liberzon, A.; Magrane, M.; Onami, J.I.; Robinson-Rechavi, M.; Sponarova, J.; Zobel,J.; et al. Quality Matters: Biocuration Experts on the Impact of Duplication and Other Data Quality Issues in Biological Databases. Genom. Proteom. Bioinform. 2020, $18,91$.

[123] Zhou, B.; Lapedriza, A.; Xiao, J.; Torralba, A.; Oliva, A. Learning Deep Features for Scene Recognition Using Places Database. In Advances in Neural Information Processing Systems, Proceedings of the 28th Annual Conference on Neural Information Processing Systems 2014 (NIPS),
[110] L. Wang, M. Peng and Q. F. Zhou, "Fall Detection Based on Convolutional Neural Networks Using Smart Insole," 2019 5th International Conference on Control, Automation and Robotics (ICCAR), Beijing, China, 2019, pp. 593-598.

[111] J. C. Ayena, L. D. Chapwouo T, M. J. -D. Otis and B. -A. J. Menelas, "An efficient home-based risk of falling assessment test based on Smartphone and instrumented insole," 2015 IEEE International Symposium on Medical Measurements and Applications (MeMeA) Proceedings, Turin, Italy, 2015, pp. 416-421.

[112] D. Chen, G. Asaeikheybari, H. Chen, W. Xu and M. -C. Huang, "Ubiquitous Fall Hazard Identification With Smart Insole," in IEEE Journal of Biomedical and Health Informatics, vol. 25, no. 7, pp. 27682776, July 2021.

[113] Zhuang Cunbo, Liu Jianhua, Xiong Hui, Ding Xiaoyu, Liu Shaoli, Weng Gang, The connotation, architecture and development trend of product digital twins, Computer Integrated Manufacturing System, 2017.4, P7530768 .

[114] Grieves M, Vickers J. Digital twin: mitigating unpredictable, undesirable emergent behavior in complex systems[M], Kahlen FJ, Flumerfelt S, Alves A. Transdisciplinary Perspectives on Complex Systems: New Findings and Approaches. Cham: Springer International Publishing, 2017: 85-113.

Montreal, QC, Canada, 8-13 December 2014; MIT Press: Cambridge, MA, USA, 2014.

[124] Gao J, Yang Y, Lin P, et al. Computer vision in healthcare applications[J]. 2018.

[125] Muhammad K, Khan S, Kumar N, et al. Vision-based personalized Wireless Capsule Endoscopy for smart healthcare: Taxonomy, literature review, opportunities and challenges[J]. Future Generation Computer Systems, 2020, 113: 266-280.

[126] J. Wu, Y. Yang, X. Cheng, H. Zuo and Z. Cheng, "The Development of Digital Twin Technology Review," 2020 Chinese Automation Congress (CAC), 2020, pp. 4901-4906, doi: 10.1109/CAC51589.2020.9327756.

[127] Ramalingame R, Hu Z, Gerlach C, et al. Shoe insole with MWCNTPDMS-composite sensors for pressure monitoring[C]//2017 IEEE SENSORS. IEEE, 2017: 1-3.

[128] Tamm T, Pärlin K, Tiimus T, et al. Smart insole sensors for sports and rehabilitation[C]//Nanosensors, Biosensors, and Info-Tech Sensors and Systems 2014. International Society for Optics and Photonics, 2014, 9060: 90600L.

[129] Dai Y, Xie Y, Chen J, et al. A lamination-based piezoelectric insole gait analysis system for massive production for Internet-of-health things[J]. International Journal of Distributed Sensor Networks, 2020, 16(3): 1550147720905431.

[130] S. Gao, J. -L. Chen, Y. -N. Dai, R. Wang, S. -B. Kang and L. -J. Xu, "Piezoelectric-Based Insole Force Sensing for Gait Analysis in the Internet of Health Things," in IEEE Consumer Electronics Magazine, vol. 10, no. 1, pp. 39-44, 1 Jan. 2021, doi: 10.1109/MCE.2020.2986828.

[131] P. Iso-Ketola et al., "Development of a Lower Extremity Rehabilitation Aid Utilizing an Insole-Integrated Load Sensor Matrix and a SoleEmbedded Measurement Node," 2009 International Symposium on Wearable Computers, 2009, pp. 107-114, doi: 10.1109/ISWC.2009.20. 